\title{
Measurement of the atmospheric muon flux with the NEMO Phase-1 detector
}

S. Aiello ${ }^{\mathrm{e}}$, F. Ameli ${ }^{\mathrm{i}}$, I. Amore ${ }^{\mathrm{a}, \ell}$, M. Anghinolfi ${ }^{\mathrm{f}}$, A. Anzalone ${ }^{\mathrm{a}}$, G. Barbarino ${ }^{\mathrm{g}, \mathrm{n}}$, M. Battaglieri ${ }^{\mathrm{f}}$, M. Bazzotti ${ }^{\mathrm{d}, \mathrm{k}}$, A. Bersanif $^{\mathrm{f}, \mathrm{m}}$, N. Beverini ${ }^{\mathrm{h}, \mathrm{o}}, \mathrm{S}_{\text {. Biagi }}^{\mathrm{d}, \mathrm{k}}$, M. Bonori ${ }^{\mathrm{i}, \mathrm{q}}$, B. Bouhadef ${ }^{\mathrm{h}, \mathrm{o}}$, M. Brunoldi ${ }^{\mathrm{m}}$, G. Cacopardo ${ }^{\mathrm{a}}$, A. Capone ${ }^{\mathrm{i}, \mathrm{q}}$, L. Caponetto ${ }^{\mathrm{e}, 3}$, G. Carminati ${ }^{\mathrm{d}, \mathrm{k}}$, T. Chiarusi $^{\mathrm{d}, \mathrm{k}}$, M. Circella $^{\mathrm{c}}$, R. Cocimano ${ }^{\mathrm{a}}$, R. Coniglione ${ }^{\mathrm{a}}$, M. Cordelli $^{\mathrm{b}}$, M. Costa $^{\mathrm{a}}$, A. D'Amico ${ }^{\text {a }}$, G. De Bonis ${ }^{\mathrm{h}, \mathrm{o}}$, C. De Marzo ${ }^{\mathrm{c}, \mathrm{j}, 1}$, G. De Rosa ${ }^{\mathrm{g}}$,

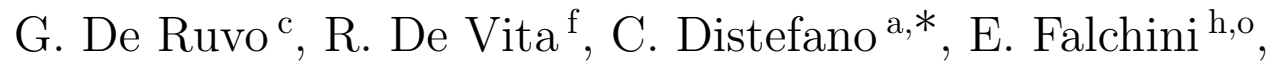
V. Flaminio ${ }^{\text {h,o }}$, K. Fratini ${ }^{\mathrm{f}}$, A. Gabriellid ${ }^{\mathrm{d}, \mathrm{k}}$, S. Galatà ${ }^{\mathrm{a}, \ell, 2}$, E. Gandolfi ${ }^{\mathrm{d}, \mathrm{k}}$, G. Giacomelli ${ }^{\mathrm{d}, \mathrm{k}}$, F. Giorgi $^{\mathrm{d}, \mathrm{k}}$, G. Giovanetti ${ }^{\mathrm{i}, \mathrm{q}}$, A. Grimaldi ${ }^{\text {e }}$, R. Habel $^{\text {b }}$, M. Imbesi ${ }^{\text {a }}$, V. Kulikovsky ${ }^{\mathrm{f}}$, D. Lattuada ${ }^{\mathrm{a}, \ell}$, E. Leonora ${ }^{\mathrm{e}, \ell}$, A. Lonardo $^{\mathrm{i}}$, D. Lo Presti $^{\mathrm{e}, \ell}$, F. Lucarelli ${ }^{\mathrm{i}, \mathrm{q}}$, A. Marinelli ${ }^{\mathrm{h}, \mathrm{o}}$, A. Margiotta ${ }^{\mathrm{d}, \mathrm{k}}$, A. Martini ${ }^{\mathrm{b}}$, R. Masullo ${ }^{\mathrm{i}, \mathrm{q}}$, E. Migneco $^{\mathrm{a}, \ell}$, S. Minutoli ${ }^{\mathrm{f}}$, M. Morganti $^{\mathrm{h}, \mathrm{o}}$, P. Musico ${ }^{\text {f }}$, M. Musumeci ${ }^{\text {a }}$, C.A. Nicolau ${ }^{\mathrm{i}}$, A. Orlando ${ }^{\mathrm{a}}$, M. Osipenko ${ }^{\text {f }}$, R. Papaleo ${ }^{a}$, V. Pappalardo ${ }^{a}$, P. Piattelli $^{\text {a }}$, D. Piombo $^{f}$, G. Raia $^{\mathrm{a}}, \mathrm{N}_{\text {. Randazzo }}{ }^{\mathrm{e}}, \mathrm{S}_{\text {. Reito }}{ }^{\mathrm{e}}$, G. Ricco $^{\mathrm{f}, \mathrm{m}}$, G. Riccobene ${ }^{\mathrm{a}}$, M. Ripani ${ }^{\mathrm{f}}$, A. Rovelli ${ }^{\mathrm{a}}$, M. Ruppi $^{\mathrm{c}, \mathrm{j}}$, G.V. Russo ${ }^{\mathrm{e}, \ell}$, S. Russo $^{\mathrm{g}, \mathrm{n}}$, P. Sapienza ${ }^{a}$, D. Sciliberto ${ }^{\mathrm{e}}$, M. Sedita ${ }^{a}$, E. Shirokov $^{r}$, F. Simeone ${ }^{\mathrm{i}, \mathrm{q}}$, V. Sipala ${ }^{\mathrm{e}, \ell}$, M. Spurio ${ }^{\mathrm{d}, \mathrm{k}}$, M. Taiuti ${ }^{\mathrm{f}, \mathrm{m}}$, L. Trasatti $^{\mathrm{b}}$, S. Urso ${ }^{\mathrm{e}}$, M. Vecchi ${ }^{\mathrm{i}, \mathrm{q}}$, P. Vicini ${ }^{\mathrm{i}}$, R. Wischnewski ${ }^{\mathrm{i}}$.

${ }^{a}$ Laboratori Nazionali del Sud INFN, Via S.Sofia 62, 95123, Catania, Italy

${ }^{\mathrm{b}}$ Laboratori Nazionali di Frascati INFN, Via Enrico Fermi 40, 00044, Frascati (RM), Italy

${ }^{\mathrm{c}}$ INFN Sezione Bari, Via Amendola 173, 70126, Bari, Italy

${ }^{\mathrm{d} I N F N}$ Sezione Bologna, V.le Berti Pichat 6-2, 40127, Bologna, Italy

e INFN Sezione Catania, Via S.Sofia 64, 95123, Catania, Italy

${ }^{\mathrm{f}}$ INFN Sezione Genova, Via Dodecaneso 33, 16146, Genova, Italy

g INFN Sezione Napoli, Via Cintia, 80126, Napoli, Italy 
${ }^{\mathrm{h}}$ INFN Sezione Pisa, Polo Fibonacci, Largo Bruno Pontecorvo 3, 56127, Pisa, Italy

${ }^{\mathrm{i} I N F N}$ Sezione Roma 1, P.le A. Moro 2, 00185, Roma, Italy

${ }^{\mathrm{j} D i p a r t i m e n t o}$ Interateneo di Fisica Università di Bari, Via Amendola 173, 70126, Bari, Italy

${ }^{\mathrm{k}}$ Dipartimento di Fisica Università di Bologna, V.le Berti Pichat 6-2, 40127, Bologna, Italy

${ }^{\ell}$ Dipartimento di Fisica e Astronomia Università di Catania, Via S.Sofia 64, 95123, Catania, Italy

${ }^{\mathrm{m}}$ Dipartimento di Fisica Università di Genova, Via Dodecaneso 33, 16146, Genova, Italy

${ }^{\mathrm{n}}$ Dipartimento di Scienze Fisiche Università di Napoli, Via Cintia, 80126, Napoli, Italy

- Dipartimento di Fisica Università di Pisa, Polo Fibonacci, Largo Bruno Pontecorvo 3, 56127, Pisa, Italy

${ }^{\mathrm{p}}$ Centro Interdisciplinare di Bioacustica e Ricerche Ambientali, Dipartimento di Biologia Animale Università di Pavia, Via Taramelli 24, 27100, Pavia, Italy

qDipartimento di Fisica Università "Sapienza", P.le A. Moro 2, 00185, Roma, Italy

${ }^{\mathrm{r}}$ Faculty of Physics, Moscow State University, 119992, Moscow, Russia

\begin{abstract}
The NEMO Collaboration installed and operated an underwater detector including prototypes of the critical elements of a possible underwater $\mathrm{km}^{3}$ neutrino telescope: a four-floor tower (called Mini-Tower) and a Junction Box. The detector was developed to test some of the main systems of the $\mathrm{km}^{3}$ detector, including the data transmission, the power distribution, the timing calibration and the acoustic positioning systems as well as to verify the capabilities of a single tridimensional detection structure to reconstruct muon tracks. We present results of the analysis of the data collected with the NEMO Mini-Tower. The position of photomultiplier tubes (PMTs) is determined through the acoustic position system. Signals detected with PMTs are used to reconstruct the tracks of atmospheric muons. The angular distribution of atmospheric muons was measured and results compared to Monte Carlo simulations.
\end{abstract}

Key words: Atmospheric muons, Neutrino telescopes, NEMO PACS: 95.55.Vj, 95.85.Ry, 96.40.Tv 


\section{Introduction}

High energy neutrinos are considered optimal probes to identify the sources of high energy cosmic rays. Many indications suggest that cosmic objects, where acceleration of charged particles takes place, should also produce high energy gamma ray and neutrino fluxes. Indeed, $p \gamma$ or $p p$ interactions responsible for $>\mathrm{TeV}$ neutrinos and $\gamma$ rays, are expected to occur in several astrophysical environments such as supernova remnants, microquasars, gamma-ray bursts and active galactic nuclei [1]. For many sources the neutrino flux, produced in the decay chains due to charge pions, is expected to be similar to the flux of high-energy gamma rays of hadronic origin, which can be measured by TeV gamma ray telescopes such as MAGIC [2], HESS [3] and VERITAS [4]. Because of the low expected neutrino fluxes from galactic and extragalactic sources [5], the effective opening of the high energy neutrino astronomy era can really be made with detectors of $\mathrm{km}^{3}$ scale. After the success of the first generation of underwater/ice neutrino telescopes, such as BAIKAL [6] and AMANDA [7], the construction of the first $\mathrm{km}^{3}$ telescope, IceCube, [8] started at the South Pole. The detector is announced to be completed by 2011. In the Mediterranean Sea, the ANTARES telescope is taking data since 2006 in a partial configuration and since 2008 in its full set-up [9]. The ANTARES Collaboration together with the NESTOR [10 and NEMO 11 Collaborations are conducting an intense $R \& D$ activity for the future $\mathrm{km}^{3}$ Mediterranean telescope. Recently the three collaborations joined their efforts to design the KM3NeT undersea infrastructure that will host a $\mathrm{km}^{3}$ telescope; its construction is expected to start by 2013 [12.

The activity of the NEMO Collaboration was mainly focused on the search and characterization of an optimal site for the detector installation and on the development of key technologies for the $\mathrm{km}^{3}$ underwater telescope to be installed in the Mediterranean Sea. A deep sea site with optimal features in terms of depth and water optical properties was identified at a depth of 3500 m about $80 \mathrm{~km}$ off-shore from Capo Passero (Southern cape of Sicily). A long term monitoring of the site has been carried out [13].

An other effort of the NEMO Collaboration was the definition of a feasibility study of the $\mathrm{km}^{3}$ detector, which included the analysis of the construction and

\footnotetext{
* Fax: +39095542398

Email address: distefano_c@lns.infn.it (C. Distefano).

1 Deceased

2 Present address, Centre de Physique des Particules de Marseille, CNRS/IN2P3 et Univ. de la Méditerranée, 163 Av. de Luminy, Case 902, 13288 Marseille Cedex 9, France

3 Present address, CNRS/IN2P3/IPNL, Domaine scientifique de la Doua, Bâtiment Paul Dirac 4, Rue Enrico Fermi, Lyon, France
} 
installation issues and the optimization of the detector geometry by means of numerical simulations.

To ensure an adequate process of validation of the proposed solutions, a technological demonstrator was built and installed off-shore the port of Catania (Sicily, Italy). This project, called NEMO Phase-1, allowed the test and the qualification of the key technological elements (mechanics, electronics, data transmission, power distribution, acoustic positioning and time calibration system) proposed for the $\mathrm{km}^{3}$ detector [14]. Some of the technical solutions, further developed within the KM3NeT Consortium, have been proposed for the $\mathrm{km}^{3}$ detector construction.

In this paper, after a detailed description of the NEMO Phase-1 lay-out and operation, we focus on the atmospheric muon data analysis procedure and present the main results. In particular, the atmospheric muon angular distribution was measured and compared with Monte Carlo simulations. The vertical muon flux was determined from the angular distribution, computing the related depth intensity relation. Results were compared with theoretical predictions and with results of other experiments.

\section{The NEMO Phase-1 detector}

The NEMO Phase-1 apparatus is composed of two main elements: the MiniTower and the Junction Box (JB) interconnected as sketched in Fig. 1 and described in the following. NEMO Phase-1 was installed between 10 and 19 December 2006 at the underwater Test Site of the Laboratori Nazionali del Sud, off shore Catania at a depth of $2080 \mathrm{~m}$, latitude: $37^{\circ} 33^{\prime} 4^{\prime \prime} \mathrm{N}$ and longitude: $15^{\circ} 23$ ' 2" E (Fig. 2). The operation was conducted with the cable layer vessel TELIRI [15]. The JB and the tower were deployed from the sea surface by means of a winch and positioned on the seabed with an accuracy of a few meters.

The JB was then connected to the main cable termination and the tower to the JB with electro-optical links equipped with wet mateable hybrid connectors. The connection operations were performed with an underwater Remotely Operated Vehicle (ROV). The operation was completed with the successful unfurling of the tower that assumed the correct configuration. All active elements, such as PMTs, electronics, acoustic positioning, data transmission and acquisition, worked correctly.

After four weeks of successful operation, two problems were encountered, which prevented a fully efficient exploitation of the apparatus. Firstly, a loss of buoyancy of the main buoy of the Mini-Tower occurred, which caused a slow 


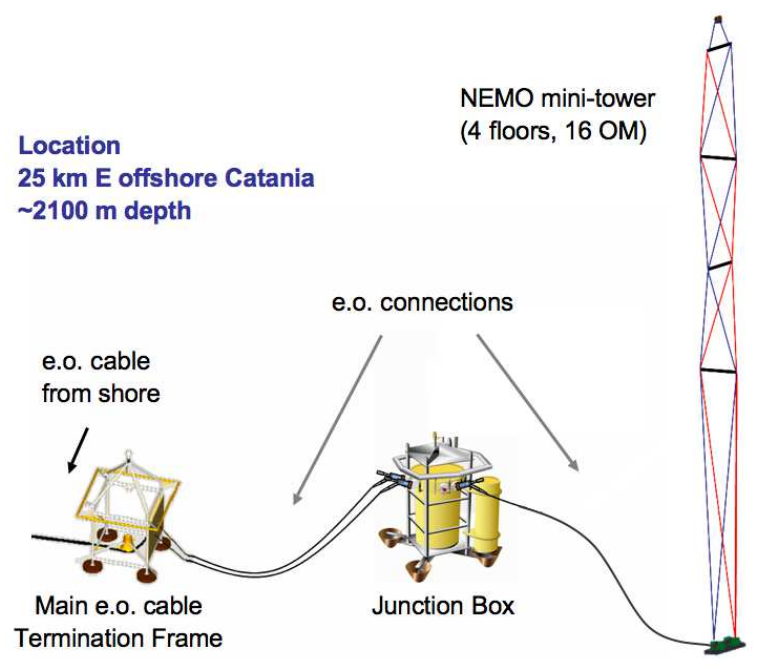

Fig. 1. Lay-out of the NEMO Phase-1 installation at the Catania Test Site.

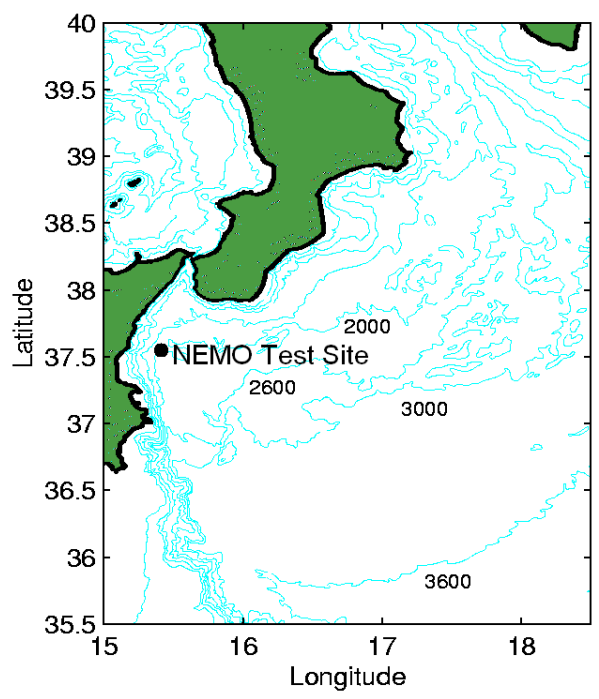

Fig. 2. Location of the Underwater Test Site of the Laboratori Nazionali del Sud.

sinking of the whole tower; this problem was later ascribed to a poor manufacturing process of the buoy. Then, about four months after the connection, the optical fiber transmission started to show a significant attenuation at the JB level. The JB was recovered in June 2007. After tests in a hyperbaric chamber, the problem was identified in a faulty optical penetrator. The problem was solved by bypassing the connector with a redesign of the optical system lay-out. The JB was re-installed in April 2008 and proved to work correctly. 
In spite of these problems, the NEMO Phase-1 allowed to validate a number of technical solutions for the $\mathrm{km}^{3}$ telescope and to demonstrate the capability of the NEMO tower to detect and track muons. During 5 months of operation about 500 GB of data were recorded.

\subsection{The Junction Box}

The JB provided connection between the main electro-optical cable and the detector structures and was designed to host and protect from the effects of corrosion and pressure the opto-electronic boards dedicated to the distribution and the control of the power supply and digitized signals.

The NEMO Phase-1 JB was built following the concept of double containment. Pressure resistant steel vessels were hosted inside a large fiberglass container, filled with silicon oil and pressure compensated. This solution has the advantage to decouple the two problems of pressure and corrosion resistance. All the electronics components that were proven able to withstand high pressure were installed directly in the oil bath [14]. The JB was equipped with 6 (2 inputs and 4 outputs) hybrid electro-optical wet mateable bulkheads. The two inputs were connected, by means of two underwater cables, to the two outputs of the termination frame of the main electro-optical cable, while one of the four available outputs of the JB was used to connect the Mini-Tower by means of a $300 \mathrm{~m}$ electro-optical link. The JB was equipped with a HV transformer and

switches to distribute power to the output connectors, and with a system of optical couplers to connect the fibers from the input to the output connectors.

\subsection{The Mini-Tower}

The Mini-Tower was a small-size prototype of the NEMO Tower [16]. It was a three dimensional flexible structure composed by a sequence of four horizontal elements (floors) interlinked by a system of tensioning ropes and anchored on the seabed. The structure was kept vertical by an appropriate buoyancy on the top.

The storey was a $15 \mathrm{~m}$ long structure supporting two Optical Modules (OMs), one down-looking and one horizontally looking, at each end: four OMs were installed on each storey. Each floor was connected to the following one by means of four ropes arranged in such a way to force each floor to a position perpendicular to its vertical neighbors. The floors were vertically spaced by $40 \mathrm{~m}$. An additional spacing of $100 \mathrm{~m}$ was present between the tower base and the lowermost floor (Fig. 33). 


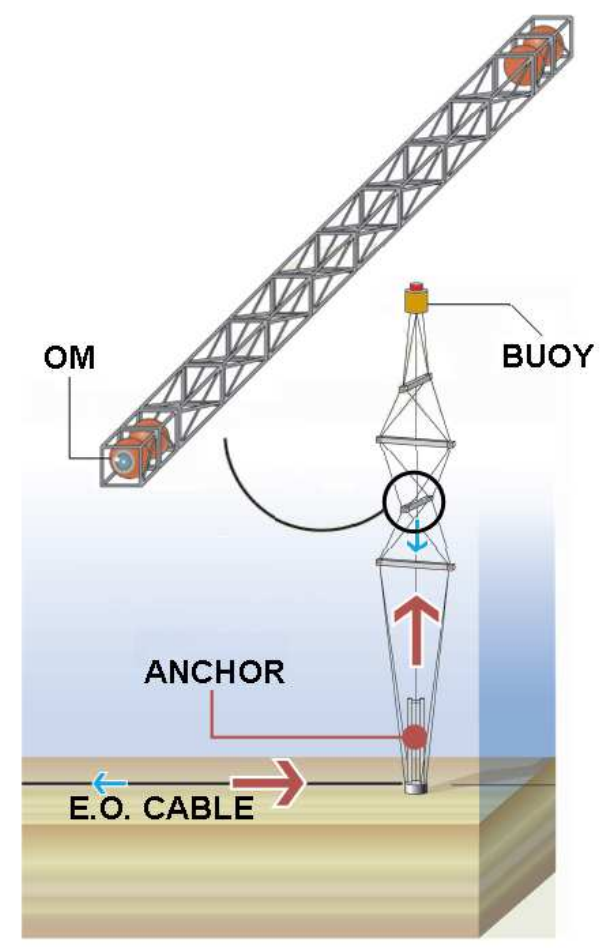

Fig. 3. Sketch of the NEMO Mini-Tower, in which 4 floors were equipped with a total of 16 PMTs (the electronics positioned along the beam is not indicated, see Fig. (4).

In addition to the 16 OMs the instrumentation installed on the Mini-Tower included several sensors for calibration and environmental monitoring: an Acoustic Doppler Current Profiler (RD Instruments Workhorse ADCP) to measure water current; a light transmissometer (Wetlabs $\mathrm{C}^{*}$ ) to measure water transparency; a Conductivity-Temperature-Depth probe (Sea-Bird Electronics 37SM microcat CTD) to monitor sea water properties; a pair of hydrophones on each floor and on the tower base for acoustic positioning. A scheme of the instrumentation is shown in Fig. 4 .

The NEMO tower was designed to be assembled in a compact configuration, also kept during the transport and the deployment, which was performed from a surface vessel by means of a winch. After the positioning on the seabed and the connection to the undersea cable network, the tower was unfurled thanks to the pull provided by the buoy. This procedure was actuated remotely from the surface vessel by means of an acoustic device. 


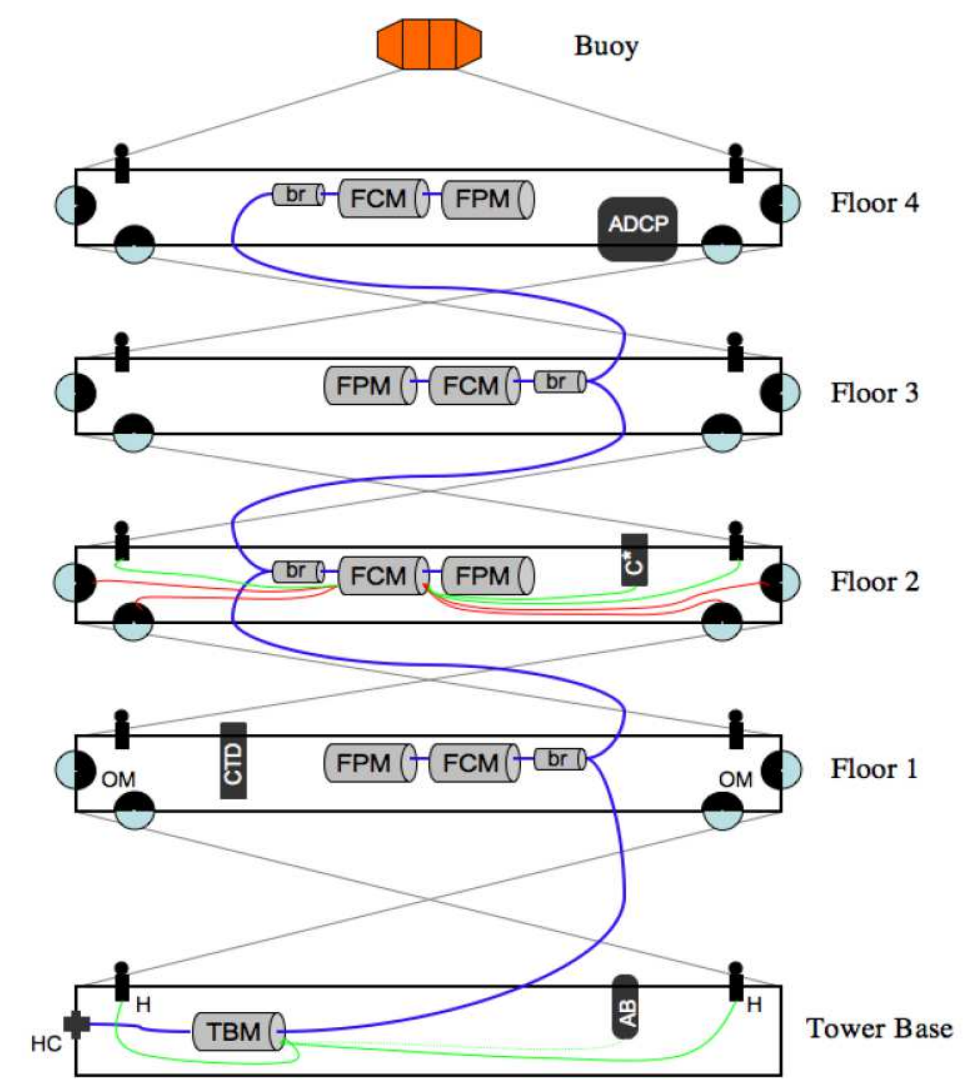

Fig. 4. Cabling layout of the NEMO Mini-Tower, including the Tower Base Module (TBM); for each of the 4 floors are indicated the floor breakouts (br), the Floor Control Modules (FCM) and the Floor Power Modules (FPM). Connection to the Junction Box is provided through a wet mateable hybrid connector (HC) on the tower base.

\subsection{The Optical Module}

Each optical module used for the NEMO Phase-1 detector was composed by a PMT enclosed in a 17" pressure resistant sphere of thick glass. The PMT was a 10" Hamamatsu R7081Sel with 10 stages [17. In spite of its large photocathode area, this PMT has a good time resolution, about 3 ns FWHM for single photoelectron pulses, with a charge resolution of $35 \%$. Mechanical and optical contact between the PMT and the internal glass surface was ensured by an optical silicon gel. A $\mu$-metal cage shielded the PMT from the Earth magnetic field. The base card circuit for the high voltage distribution (Iseg PHQ 7081SEL) required only a low voltage supply (+5 VDC) and generated all necessary voltages for cathode, grid and dynodes with a power consumption of less than $150 \mathrm{~mW}$.

A Front-end Electronics Module (FEM), built with discrete components [18], 
was also placed inside the OM. The FEM performed sampling at $200 \mathrm{MHz}$ by means of two $100 \mathrm{MHz}$ staggered Flash ADCs, whose outputs were "captured" by a Field Programmable Gate Array (FPGA). The FPGA performed threshold discrimination, stored it with an event time stamp in an internal 16 kbit FIFO, packed OM data and local slow control information and coded everything into a bit stream frame ready to be transmitted on a twisted pair at $20 \mathrm{Mb} / \mathrm{s}$. The main features of this solution are the moderate power consumption, the high resolution and the large input dynamics range obtained by a quasi-logarithmic analog compression circuit, with a raw time resolution of $5 \mathrm{~ns}$. The FEM board digitized and sent pulse waveform information up to a maximum pulse rate of $\sim 150 \mathrm{kHz}$. The FEM board was also equipped with analog and digital electronics to control the OM power supply and to monitor the operating conditions, such of temperature, humidity and electrical parameters. It could also perform a calibration of the non linear response of the logarithmic compressor.

In addition, the board provided an estimate of the average count rate by counting the number of hits with amplitude exceeding a threshold of 0.3 single photo-electron (s.p.e.) in a $10 \mathrm{~ms}$ time window as shown in sec. 3.2. This estimate did not suffer from the limitation of the data transfer process and allowed to measure the signal rate up to $6.5 \mathrm{MHz}$.

\subsection{The data acquisition system}

The design of the data acquisition system for NEMO Phase-1 was based on technical choices that allow scalability to a much larger apparatus [19,20].

The optical connection between the counting room facilities on-shore and the detector under water was driven by pairs of twin electronic boards, called Floor Control Module (FCM), located at both extremities of each optical link, and deputed to manage either the OM data stream, from off-shore to on-shore, and the Slow Control commands, sent in the opposite direction. Each FCM board was powered by a Floor Power Module (FPM) lodged on the corresponding floor, and was connected to the Tower Base Module (TBM) through an optical fiber backbone. The TBM was connected through an inter-link cable to the JB. A detailed description of the Mini-Tower electronics is given in [20].

The OM data acquisition worked as follows: each off-shore FCM multiplexed the signal produced by the corresponding four OM-FEM pairs in a floor, converted them from electric to optical and sent it on-shore through the optical link. Each on-shore FCM, hosted on a dedicated server, de-multiplexed the incoming data and distributed it to the Trigger and Data Acquisition System, which was composed of the MasterCPU server, for data filtering, and a post- 
trigger data storage facility. Each on-shore server was connected to the others via a standard 1 Gigabit Ethernet network.

In order to reconstruct muon tracks using the Cerenkov technique, a common timing must be known in the whole apparatus at the level of each detection device to allow time correlation of events. For this reason a synchronous and fixed latency protocol, which embedded data and clock timing in the same serial bit stream, was used for communications between onshore and offshore. The implemented system relied on Dense Wavelength Division Multiplex (DWDM) techniques, using totally passive components with the only exception of the line termination devices, i.e. electro-optical transceivers. The precision of the common timing of the apparatus was measured and monitored using a dedicated time calibration system, as illustrated in the next subsection.

\subsection{The time calibration system}

The time calibration was performed with an embedded system to track the possible drifts of the time offsets during the operations of the apparatus underwater [21]. This system measured the offsets with which the local time counters inside the optical modules were reset on reception of the reset commands broadcasted from shore. The operation was performed with a redundant system: (1) a two-step procedure for measuring the offsets in the time measurements of the optical sensors and (2) an all-optical procedure for measuring the differences in the time offsets of the different optical modules.

In the first system the needed measurements were performed in two separate steps: using an "echo" timing calibration and using an "optical" timing calibration. The former allowed the measurement of the time delay for the signal propagation from the shore to the FCM of each floor; the latter, based on a network of optical fibres which distributes calibration signals from fast light

pulsers to the OMs, allowed to determine the time offsets between the FCM and each optical module connected to it.

The second system was an extension of the optical timing calibration system, which allowed to simultaneously calibrate the optical modules of different floors of the tower.

\subsection{The acoustic positioning system}

Another key requirement for the muon tracking with an underwater Cherenkov apparatus is the knowledge of each optical sensor position. While the position and orientation of the tower base was fixed and known from its installation, 
the rest of the structure could bend under the influence of sea currents. A precise determination of the position of each tower floor was achieved by means of triangulations performed by measuring the propagation times of acoustic signals between a Long-Base-Line (LBL) of acoustic beacons, placed on the sea floor, and a couple of hydrophones (labeled H0 and H1) installed on each tower floor close to the positions of the optical modules. The inclination and orientation of each tower floor was also measured by a tiltmeter-compass board placed inside the FCM.

The LBL was realized with four stand-alone battery-powered acoustic beacons and one additional beacon located on the tower base (Fig. 5). To recognize

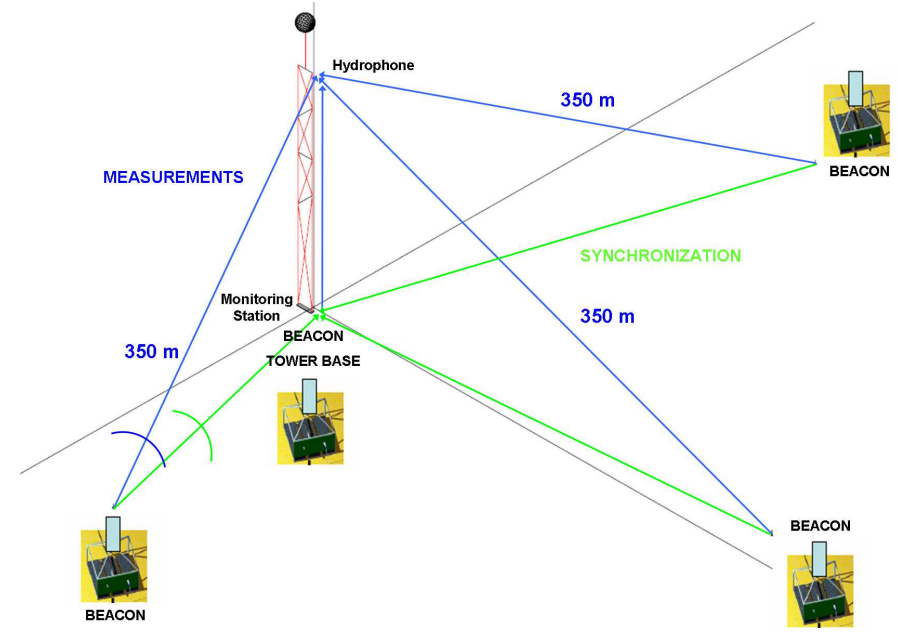

Fig. 5. Schematic view of the Acoustic Positioning System (APS) and of the Long-Base-Line (LBL) configuration.

the beacon pulses a technique called Time Spectral Spread Codes (TSSC) was used. Each beacon transmitted a unique pattern of 6 pseudo-random pulses (spaced by $1 \mathrm{~s}$ ). The duration of each pulse was $5 \mathrm{~ms}$ and the sequence of pulses was built to avoid overlap between consecutive pulses. In this way a typical beacon pulse sequence was recognized without ambiguity and all the beacons could transmit their characteristic pulse sequence at the same acoustic frequency. This was an advantage since all beacons could be identical except for the software configuration that defines the pulse sequence; the receivers were then sensitive to the $32 \mathrm{kHz}$ acoustic channel. Since the beacon clocks were autonomous, in order to determine the position of the hydrophones, the LBL had to be synchronized to the master clock of the apparatus. This synchronisation took place acoustically using a monitoring station, formed by two hydrophones, connected to the Mini-Tower data acquisition system, placed in correspondence to the tower base. Prior to the connection of the MiniTower, the four acoustic beacons providing the LBL were deployed around the apparatus at an approximate distance of $250 \mathrm{~m}$ from the tower base. In order to obtain the required accuracy of $\$ 15 \mathrm{~cm}$ - comparable with the size of the PMT - the time of flight of acoustic signals between the LBL 
beacons and the monitoring station had to be evaluated with an accuracy of the order of $10^{-4}$ sec. To achieve this goal an accurate calibration of the LBL was performed, taking into account the clock drift of the stand-alone beacons. In particular, the absolute positions of the beacons and their relative distances were determined, acoustically, at the time of detector installation, using a ROV equipped with a $32 \mathrm{kHz}$, GPS synchronized pinger and with a high accuracy pressure sensor.

\section{Control and environmental parameters}

All the Slow Control data (including data from all environmental sensors and the acoustic positioning system) were managed from shore by means of a dedicated Slow Control Management System [22] and analyzed as described in the following.

\subsection{Acoustic positioning data}

The LBL calibration procedure allowed the determination of distances between the beacons and the monitoring station, and to calculate the time of flight of the acoustic pulses as the difference between the time of arrival of the acoustic signal on each hydrophone and the time of emission of the beacon. The sound velocity was calculated using the CTD data. The time of emission of the beacon pulse, in the common detector clock reference time, was obtained measuring the time of arrival of this pulse at the monitoring station. This procedure allowed to compensate for the clock drift of the stand-alone beacons (about $20 \mathrm{~ns} / \mathrm{s}$ ) during the livetime of the apparatus. In order to merge in postprocessing the acoustic positions data together with optical module detection information, both were time stamped with a universal time reference tag.

The acoustic positioning system data were extensively analyzed. The tower positions were reconstructed and the movements were measured as a function of time, on long and short time scales [23].

In order to estimate the accuracy of the positioning system, the distances between hydrophones $\mathrm{H} 0$ and $\mathrm{H} 1$ on the same floor were measured. In Fig.6 the distance H0-H1 measured for floor 2 is shown. This result indicates that the obtained accuracy in the determination of hydrophone positions is better than $10 \mathrm{~cm}$. 


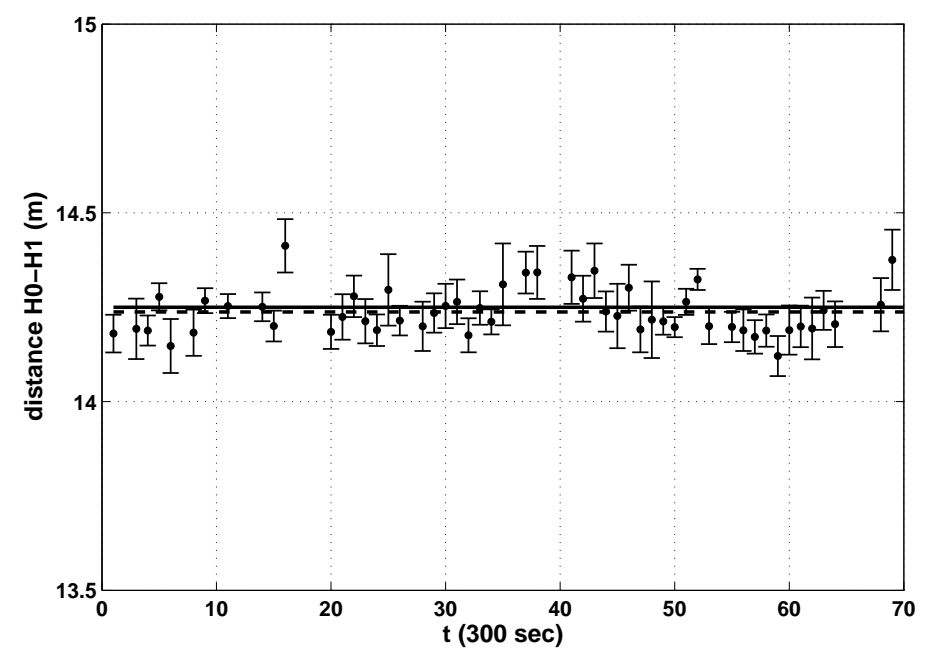

Fig. 6. Distance H0-H1 measured for floor 2. Each point is the averaged distance over a period of 5 minutes, in the time interval from $1^{\text {st }}$ February h.17 to $1^{\text {st }}$ February h.23 (6 hours). The mean value of the measured distance is $14.24 \pm 0.06 \mathrm{~m}$ (dashed line). This value is compared with the construction distance of $14.25 \pm 0.01 \mathrm{~m}$ (solid line), measured on-shore, during the tower integration.

\subsection{PMT counting rates}

The instantaneous PMT rate values were computed by the FEMs as described in sec. 2.3. The PMT counting rates gave an instantaneous estimate of the optical background during the detector livetime.

In Fig. 7 the histogram of the rate distribution is plotted for a PMT located on the $4^{\text {th }}$ floor, in the time interval between 10 and 20 January 2007. The histogram shows a peak in the $75-80 \mathrm{kHz}$ interval due to ${ }^{40} \mathrm{~K}$ decay plus a contribution due to diffuse bioluminescence [24]. The frequency value at the peak, commonly called the baseline of the optical background [25], was determined fitting the peak of the distribution with a Gaussian function. The baseline obtained from the fit is $72.5 \pm 3.6 \mathrm{kHz}$. The distribution, plotted in Fig. 17, shows also a tail extending to several hundreds $\mathrm{kHz}$ due to intense bioluminescence bursts. This contribution is measured by means of the socalled burst fraction and it was calculated, for comparison with the ANTARES detector data in two different ways [25]:

- the percentage of time in which the rate exceeds $200 \mathrm{kHz}$;

- the percentage of time in which the rate exceeds 1.2 times the baseline rate value.

For the data shown in Fig. 17, the percentage of time with rates exceeding 200 $\mathrm{kHz}$ was $0.3 \%$ while the percentage of time with rates larger than 1.2 times 
the baseline rate was $2.6 \%$.

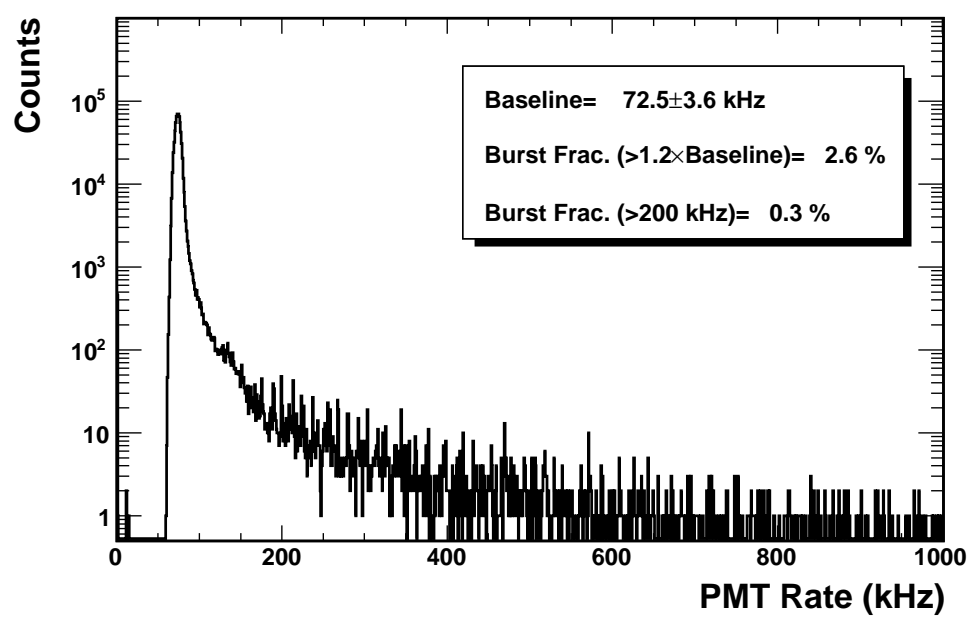

Fig. 7. Histogram of hit rate distribution from one PMT located on the $4^{\text {th }}$ floor, recorded in the period 10-20 January 2007.

The baseline and burst fraction of the optical background were determined for the full period of the detector operation. Typical results, for an OM located in the $4^{\text {th }}$ Mini-Tower floor, are shown in Fig. 8. The data indicate two time intervals with different characteristics of the optical background. The first period, from the detector activation to the first week of February 2007, was characterized by a baseline rate of $\sim 73 \mathrm{kHz}$ and a burst fraction of the order of a few percents (with peaks up to $20 \%$ ) while the second one, starting approximately on February 11, characterized by a slightly lower baseline rate (average $\sim 67 \mathrm{kHz}$ ) and a higher burst fraction $(\sim 20 \%)$. These results can be explained as due to two effects: a change of the water properties and a change in the detector configuration due to the loss of buoyancy of the tower. Starting from February 2007, in fact, the detector fell down by about 40 meters; thus the $4^{\text {th }}$ floor was at about 80 meters from the sea bottom. On the other hand during the same period of time we observed an increase of the underwater currents [23], and as shown by the ANTARES Collaboration [26], the level of bioluminescence is usually correlated with the value of the sea current. The somehow contradictory decrease of the baseline can be explained as a consequence of the increase of water turbidity, due to particulate, close to the bottom and under the effect of the increased current.

\section{Atmospheric muon analysis}

In order to reconstruct atmospheric muon tracks and to measure the muon flux a dedicated data selection and analysis procedure was applied. The atmospheric muon track analysis was implemented for a limited period of the 



Fig. 8. Time dependence of the baseline rate (upper panel) and of the burst fraction (lower panel) for a PMT located on the $4^{\text {th }}$ floor in the period from 7 January (07/01/07) to 31 March 2007 (07/03/31). The shaded areas represent the two periods of data acquisition when the muon trigger was active, see text.

detector livetime, shown by the shaded area in Fig. 8: from January 23 and 242007 -when the tower was completely unfolded- and from March 2 to April 12 2007, when the two lowest floors of the tower were laying on the seabed.

\subsection{The on-line muon trigger}

As described in section 2.4, the data acquired by the OMs were distributed, onshore, to the MasterCPU. On this machine, an on-line data selection algorithm was implemented to select muon events among the optical background.

Since the main contribution to optical noise was due to uncorrelated PMTs hits -with an average rate of about $70 \mathrm{kHz}$ - the selection criterion (hereafter trigger seed) consisted in imposing time coincidence in a narrow time-window of 20 ns among hits occurred on pairs of adjacent PMTs (i.e. the two PMTs located at the same extremity of the same floor). This trigger seed is called Simple Coincidence (SC).

When an SC trigger occurred, the data acquired by all the PMTs within a Triggered Time Window (TTW), centered around the SC time, were stored on file and identified as possible muon event for off-line data analysis. If another trigger seed occurred in the TTW after the first one, the TTW itself was extended by $\Delta t_{T T W}$ after the new seed time. 
In order to test the on-line selection algorithm, two different lengths of the TTW were used, i.e. $\Delta t_{T T W}= \pm 2 \mu$ s and $\pm 5 \mu$ s, around the trigger seed time. It was found that, after the application of the causality filter on PMT hits (see sec. 4.4), the two data sets taken with different $\Delta t_{T T W}$, were equivalent.

The measured total SC trigger rate ranged between 1.5 and $2 \mathrm{kHz}$, while the expected atmospheric muon signal in the Mini-Tower, evaluated with Monte Carlo simulations, was $\sim 1 \mathrm{~Hz}$ (see sec. 5). Therefore, a further off-line data selection, with more complex and more selective algorithms, too slow for the requirements of the on-line trigger, was necessary.

\subsection{The off-line PMT data calibration}

The first step of the off-line event analysis was PMT data calibration. PMT hits, recorded on each event file, were decompressed and calibrated [27]. First, the PMT hit wave-form was re-sampled at $2 \mathrm{GHz}$ (the ADC sampling is 200 $\mathrm{MHz}$ ). Therefore, the data values measured in ADC channels were decompressed and converted into amplitudes (in $\mathrm{mV}$ unit), using a decompression table generated during the FEM characterization phase. The rising edge of the waveform was then fitted with a sigmoid function and the hit time was evaluated at the inflection point, and then corrected with the time offset provided etc.. At the end of this process the PMT hit waveform was reconstructed: the integral charge was determined with an uncertainty of $\sigma \sim 0.3 \mathrm{pC}$; this value was converted in units of p.e. taking into account that 1 p.e. $=8$ pC. The PMT hit calibration allowed to obtain hit time evaluation with a precision of $\sigma \sim 1$ ns, i.e. 5 times better than at the raw data level.

\subsection{The off-line muon trigger}

After the PMT off-line calibration, thanks to the better time accuracy of PMT hits, the SC trigger were re-applied to reject the false coincidences found by the less accurate on-line trigger algorithm. Besides the SC trigger seed, other trigger seeds were searched in each candidate muon event: the Floor Coincidence (FC) seed, that is a coincidence between 2 hits recorded at the opposite ends of the same storey $\left(\Delta T_{F C} \leq 200 \mathrm{~ns}\right)$ and the Charge Shooting (CS) seed, that is a hit exceeding a charge threshold of 2.5 p.e..

The PMT hits of the event which satisfied at least one trigger seed were, then, selected. Among this sub-sample, the number of space-time causality relations $\left(N_{\text {Caus }}\right)$ between "trigger hits" was calculated, using the following criterion:

$$
|d t|<d r / v+20 \mathrm{~ns}
$$


where $|d t|$ is the absolute value of the time delay between two hits, $d r$ is the distance between the two corresponding PMTs (calculated using the Acoustic Positioning System data) and $v$ is the group velocity of light in seawater. The value of $N_{\text {Caus }}$ was used to reject events containing only background hits. Only the events having $N_{\text {Caus }} \geq 4$ were considered for further analysis, according to results of Monte Carlo simulations (see sec. 5 ). For the events passing this selection procedure, all PMTs hits were re-considered in the following steps of the analysis.

In Fig. 9 is shown the correlation between the number of FC and CS triggers for each event passing the selection procedure. In the left-hand side the number of selected events is plotted as a function of number of CS and FC triggers per event. In the right-hand side, the number of hits, in the whole data sample, participating in both an FC and in a CS trigger is shown. A large fraction of the selected hits, do not satisfy the two trigger conditions simultaneously.
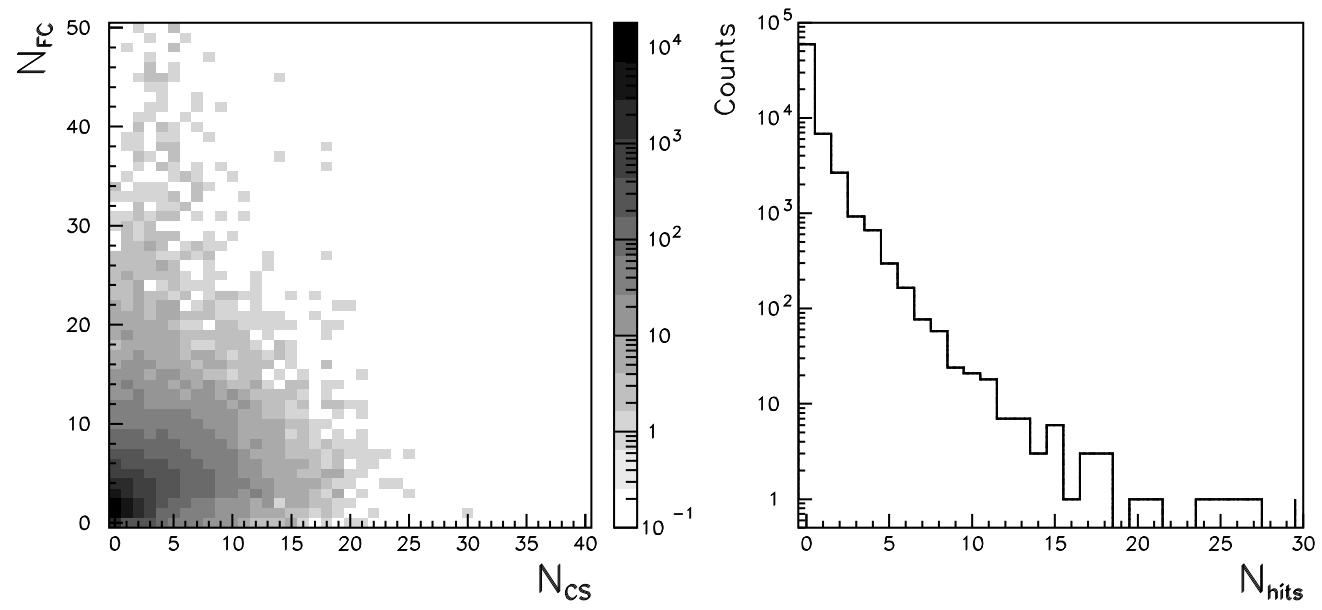

Fig. 9. Left: Contour plot showing the number of events as a function of FC and CS trigger per event. Right: Number of hits participating both in an FC and in a CS trigger (see text).

\subsection{Muon track reconstruction}

Once a muon event was identified, it was necessary to reject the hits due to the optical background before starting the muon track reconstruction procedure. First, PMT hits with amplitude smaller than 0.5 p.e. were rejected. Then, a new background filter algorithm [28, was applied, as described in the following. The $N$ PMT hits of the selected event, were sorted by arrival time, and 
the event average hit rate was calculated as

$$
f=\frac{N}{T_{N}-T_{1}}
$$

where $T_{1}$ and $T_{N}$ are the occurrence time of the "earliest" and the "latest" hits in the selected event, respectively. PMT hits were, then, grouped in samples of $n=5$ consecutive hits $\left(\right.$ hit $_{1}:$ hit $_{5}$, hit $_{2}:$ hit $_{6}, \ldots$, hit $_{N-5}:$ hit $_{N}$ ). The hit rate of each sample $f_{\text {sample }}=n / \Delta t$, where $\Delta t=T_{k+n}-T_{k}$, was calculated. The sample showing the highest hit rate is most likely to contain muon hits, and it was taken as the reference sample for the following steps of the muon reconstruction procedure. The efficiency of this algorithm was proven via Monte Carlo simulations (see sec. 5). As an example Fig. 10 shows the distribution of hit arrival times for a simulated (left-hand side) and for a real (right-hand side) muon event crossing the Mini-Tower detector. For the simulated event, the white filled histogram represents the muon-induced Čerenkov photons hits, while the black filled histogram refers to optical background hits. For the real event PMT hits are shown as a white-filled histogram. In both cases the upper plot shows the sample hit rate $f_{\text {sample }}$ with respect to the average event hit rate $f$.
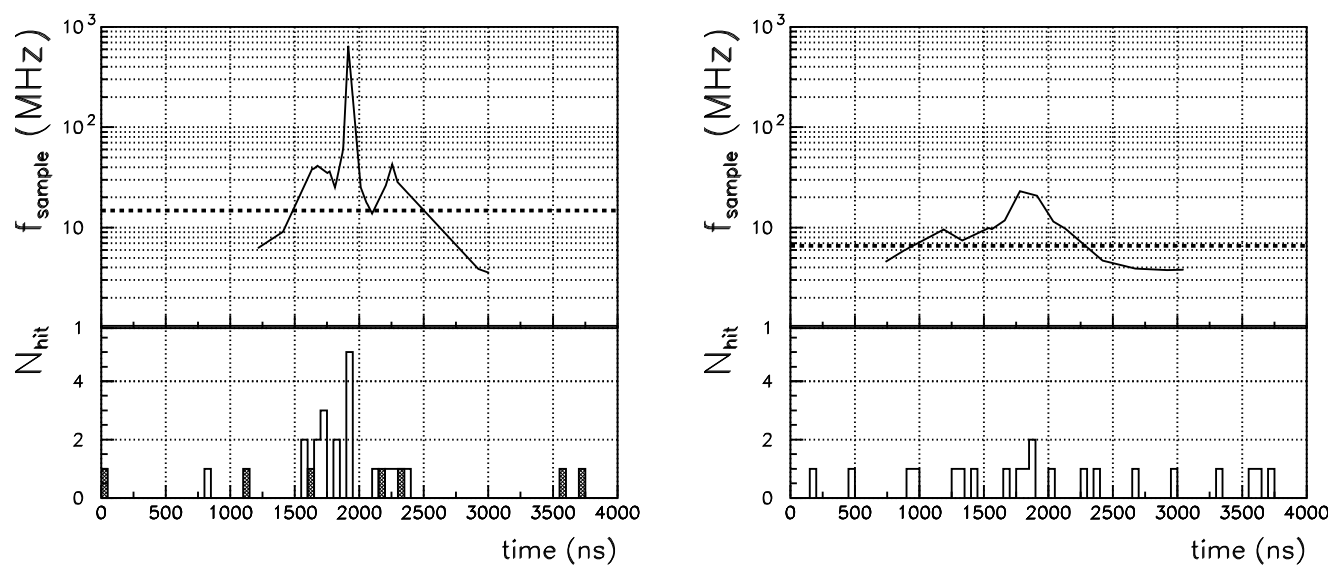

Fig. 10. Sample hit rate for $n=5$ as a function of time for a simulated muon event (left) and for an event in the data set (right). The dashed line represents the event average hit rate. The histograms in the left-hand figure represent the distribution of muon hits (white filled) and background hits (black filled). In the right-hand figure the histogram represents real PMT hits (white filled).

The following step was to apply the causality relation in Eq. 1 between each of the $n$ hits of the reference sample, and the other $N-1$ PMT hits in the event. A reference hit, causally correlated with the largest number of the other event hits, was chosen, and the ensemble formed by all the causally correlated hits was stored and used to reconstruct the atmospheric muon track. If this 
procedure preserved 6 hits, at least, the muon track direction reconstruction codes were run.

The muon direction reconstruction algorithm used in this work was originally developed by the ANTARES Collaboration and was then adapated to the NEMO tower configuration [2930]. It is a track fitting procedure based on a maximum likelihood method, that takes into account the Čerenkov light features and the possible presence of non-rejected background hits. The reconstruction strategy was applied in the way described below.

After the previous analysis cuts, hits participating to the trigger seeds could have been rejected, therefore the actual number of hits participating to the CS and CS trigger seeds was recalculated. These hits form the new ensemble on which a linear track pre-fit was applied. At least three hits were required to compute the pre-fit. The results of the pre-fit were then used as starting condition for the following fit algorithms (based on maximum likelihood method), that use all the hits in the stored sample, and surviving the optical background filter. During the likelihood fitting procedure other hits can be rejected and, since the minimum number of hits required for track fitting is $N_{\text {hit }} \geq 6$, this reduces the total number of reconstructed event 4 . Among all the tracks reconstructed with the likelihood method algorithm, only the ones satisfying the likelihood $(L)$ condition:

$$
\Lambda \equiv \frac{\log _{10}(L)}{N_{\mathrm{DOF}}}>-10
$$

were selected, according to results of Monte Carlo simulations, described in the next section.

\section{Results}

In Tab. 1 the results of the muon reconstruction procedure, at different stages of the analysis and of the reconstruction procedure, are given. The table refers to the sample of data recorded on 23-24 January 2007, shown in Fig. 8, when the tower was completely unfurled. The cuts applied on the data, at the level of the background filter and likelihood fit parameter, where chosen according to the results of the Monte Carlo simulation described below, optimized for the data taken in the period 23-24 January 2007.

$\overline{4}$ It is worth to mention that the applied likelihood reconstruction procedure is optimised for the larger size ANTARES detector 
Table 1

Results of the data analysis and track reconstruction procedure: number of events and reconstructed tracks for each step of the analysis applied in sequence (see text).

\begin{tabular}{llc}
\hline LiveTime & & 11.31 hours \\
On-line Trigger & at least $1 \mathrm{SC}$ & $6 \cdot 10^{7}$ events \\
Off-line Trigger & $N_{\text {Caus }} \geq 4$ & 465386 events \\
Background Filter & $N_{\text {hit }} \geq 6$ & 70913 events \\
Prefit reconstructed & $N_{\text {hit }} \geq 3($ hit $\in$ SC or CS $)$ & 13205 tracks \\
Likelihood reconstructed & & 3049 tracks \\
Selected & $\Lambda>-10$ & 1139 tracks \\
\hline
\end{tabular}

After the analysis cuts a total of 3049 atmospheric muon events was reconstructed with an average muon reconstruction rate of $0.075 \mathrm{~Hz}$. The limited detector size and the short livetime did not allow the detection of up-going atmospheric neutrino events, whose rate is about $10^{-6}$ times less than for the muons.

For the data recorded during the period 2 March - 12 April 2007 the acquisition livetime was 174.1 hours and the total number of reconstructed atmospheric muons was 27699, thus the average reconstruction rate was $0.044 \mathrm{~Hz}$ [31]. The lower rate of reconstructed tracks was due to the smaller number of PMTs participating in the muon reconstruction procedure, caused by the improper tower configuration (see sec. 2). These data were not used for further analysis.

\subsection{Detector Monte Carlo simulation}

In order to evaluate the detector response to atmospheric muons and compare it with the results of the data analysis, a Monte Carlo simulation was performed. Atmospheric muon events were simulated using the MUPAGE code [32], a muon event generator based on parametric formulas [33]. Atmospheric muons were generated on the surface of a can-shaped volume of water of 194 $\mathrm{m}$ height and $238 \mathrm{~m}$ radius (containing the detector) in an energy range from $20 \mathrm{GeV}$ to $500 \mathrm{TeV}$, according to the MUPAGE limits. This energy range was suitable due to the expected detector threshold and limited size. A total of $4 \cdot 10^{7}$ atmospheric muons was simulated, corresponding to the real acquisition detector livetime of 11.3 hours. The generated muon events were propagated inside the detector, using the simulation tools developed by the ANTARES Collaboration [34. These codes simulate the emission and propagation of Cerenkov light induced by muons and their secondary products (e.g. showers and $\delta$-rays), then record photo-electron signals on PMTs. As 
mentioned before, the actual run conditions of the detector were taken into account. The detector geometry was simulated using the PMT positions reconstructed by the acoustic positioning system data. The light absorption length as a function of photon wavelength was introduced, according to the one measured at the detector site [13. Once the muon PMT hits -produced by Cerenkov photons originated along the muon track- were simulated, the spurious PMT hits -due to the underwater optical noise- were introduced in the simulation. It was assumed that the optical background produces uncorrelated s.p.e. signals in the PMTs. In the present work, optical background hits were simulated, for each PMT, according to the real counting rate spectrum measured during the selected period of detector operation (e.g. see Fig. 7). The Mini-Tower DAQ electronics and the on-line trigger were also simulated.

Monte Carlo events surviving the on-line trigger simulation, were processed using the same analysis chain used for the detector data analysis. In Fig. 11 the rate of reconstructed tracks as a function of number of PMT hits, after the causality filter, is shown both for Monte Carlo events and detector data. Only the events preserving at least 6 PMT hits could be processed by the track reconstruction algorithms.

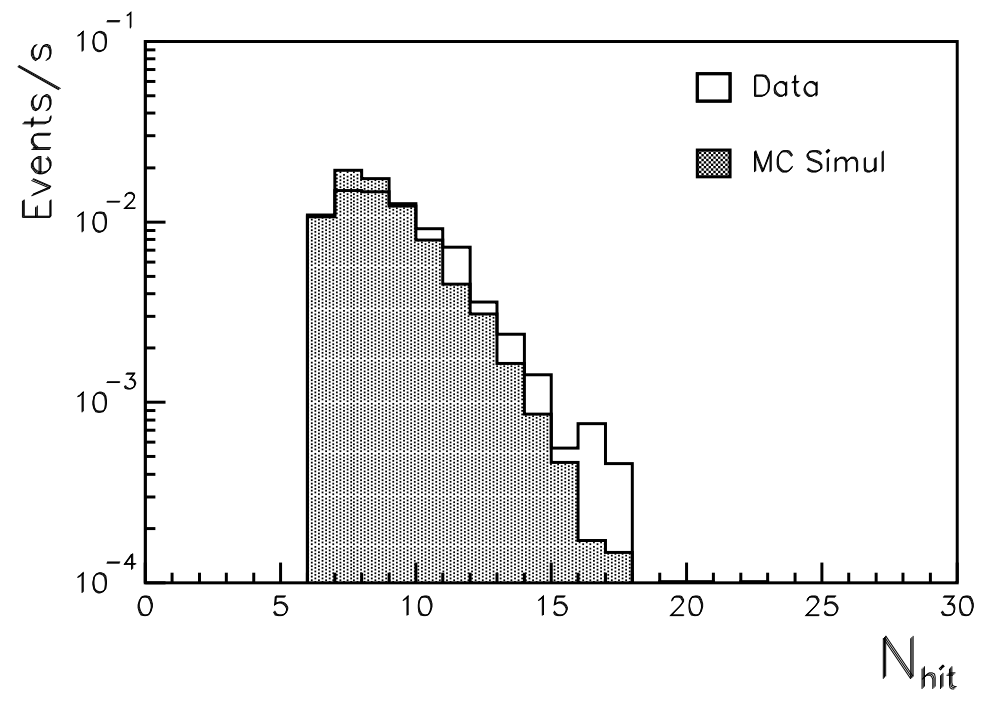

Fig. 11. Rate of muon tracks, as a function of the number of PMT hits, for events reconstructed from the data or from simulations, and Monte Carlo events.

\subsection{Comparison between data and simulations}

The good agreement found between data and Monte Carlo events is shown in Fig. 12. The likelihood distribution of the reconstructed muon track rate is 
shown both for data and Monte Carlo events.

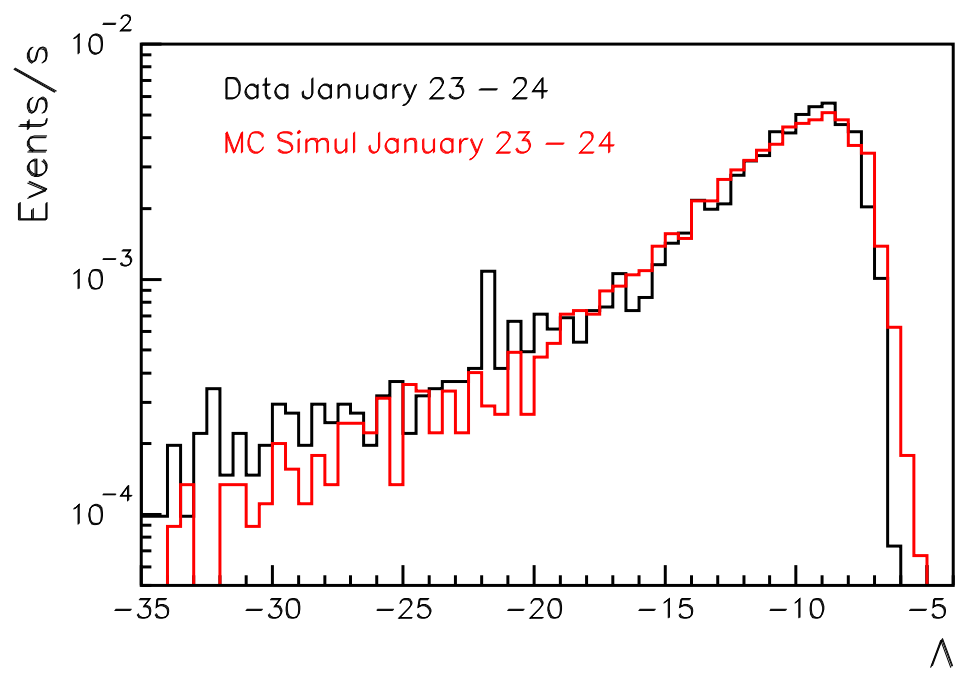

Fig. 12. Likelihood spectra of reconstructed muon tracks.

Among the reconstructed tracks, only the ones with a fit quality parameter $\Lambda>-10$ were selected. In Fig. 13 the zenith angular distribution of the reconstructed muon track rate, after the fit quality cut, is shown. The same distribution for Monte Carlo events is shown for comparison. The KolmogorovSmirnov test [35] was performed 5 to quantitatively evaluate the agreement between data and Monte Carlo events, for the distributions plotted in Fig. 13 . The test probability was found to be 0.81 , proving a good agreement between the two distributions.

\subsection{Depth Intensity Relation for Atmospheric Muons}

The final step of the analysis was the calculation of the so called Depth Intensity Relation for the reconstructed atmospheric muon tracks, that is the measurement of the vertical muon flux intensity as a function of the muon slant depth in water. An atmospheric muon, reaching the detector located at a depth $D$ from a Zenith angle $\vartheta_{Z}$, propagates through a water slant $h$

$$
h=\frac{D}{\cos \vartheta_{Z}} .
$$

The Depth Intensity Relation, thus, gives an estimate of the vertical muon intensity as a function of the equivalent water depth $h$ [36].

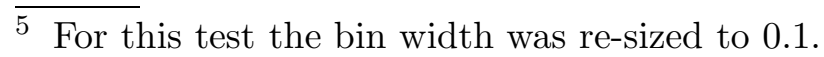






Fig. 13. Angular distributions of reconstructed muon track rate after applying the likelihood quality cut $(\Lambda>-10)$.

The muon intensity $I\left(\vartheta_{\mu}\right)$ as a function of the muon direction $\vartheta_{\mu}$, was calculated using the relation

$$
I\left(\vartheta_{\mu}\right)=\frac{N_{\mu}\left(\vartheta_{\mu}\right) \cdot m\left(\vartheta_{\mu}\right)}{A_{e f f}\left(\vartheta_{\mu}\right) \cdot T \cdot \Delta \Omega}
$$

where

- $N_{\mu}\left(\vartheta_{\mu}\right)$ is the number of muon events assigned by the analysis to the angular interval centered around $\cos \vartheta_{\mu}$. For this, we considered the angular distribution $N_{\mu}\left(\vartheta_{\mu}^{r e c}\right)$ obtained from the reconstruction, without applying cuts. This distribution appears smeared because of the detector angular resolution. We applied an iterative unfolding method based on Bayes' theorem [37];

- $m\left(\vartheta_{\mu}\right)$ is the mean muon multiplicity at the angle $\vartheta_{\mu}$ and at the detector depth. It was determined from the Monte Carlo simulations and it is shown in Fig. 14;

- $A_{\text {eff }}\left(\vartheta_{\mu}\right)$ is the reconstruction effective area at the muon angle $\vartheta_{\mu}$, determined by Monte Carlo simulations and shown in Fig. 15.

- $T$ is the data livetime. We used only the data acquired in the period on 23-24 January 2007 with $T=11.3$ hours;

- $\Delta \Omega$ is the solid angle covered by the corresponding $\cos \vartheta_{\mu}$ interval.

Fig. 16 shows the angular distribution of the atmospheric muon flux obtained from Eq. 5. Error bars include both statistical and systematic uncertainties 


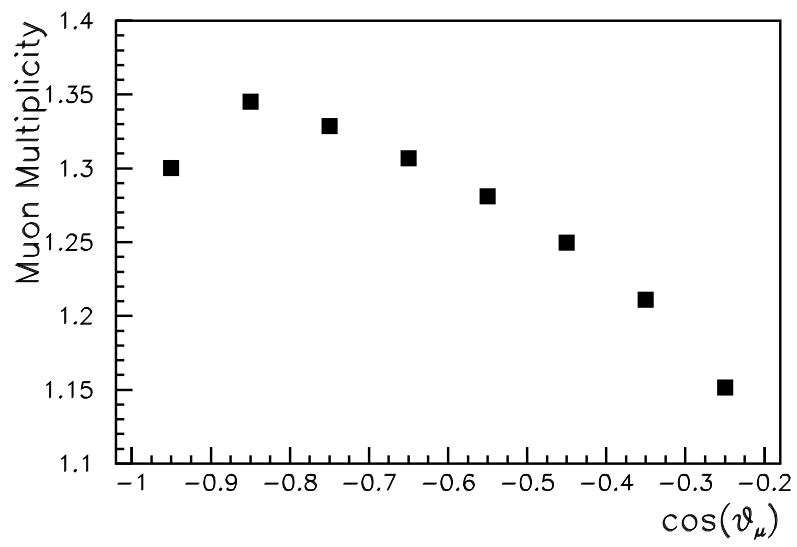

Fig. 14. Mean muon multiplicity as a function of the angle $\vartheta_{\mu}$ and at the detector depth.

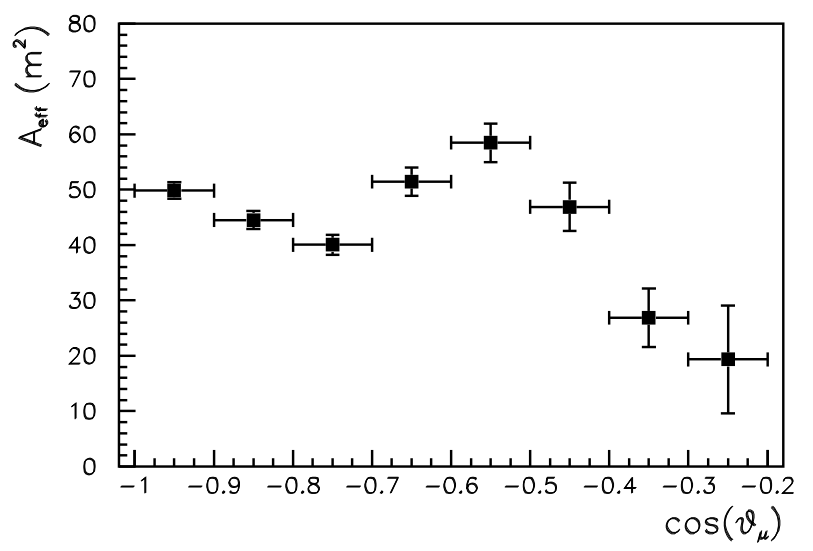

Fig. 15. Reconstruction effective area as a function of the muon angle $\vartheta_{\mu}$, determined by Monte Carlo simulations.

added in quadrature. Systematic errors were evaluated via Monte Carlo simulation, taking into account the uncertainties on the input parameters: the light absorption length in water $\left(L_{a}\right)$, the light scattering length in water $\left(L_{b}\right)$, the PMT quantum efficiency and the angular acceptance of the Optical Module [17,38]. In Tab. 2 the contributions of the different parameters to the total systematic error are reported. The systematic error is mainly a scale error common to all measured points; its contribution to the point to point error is considerably smaller. 


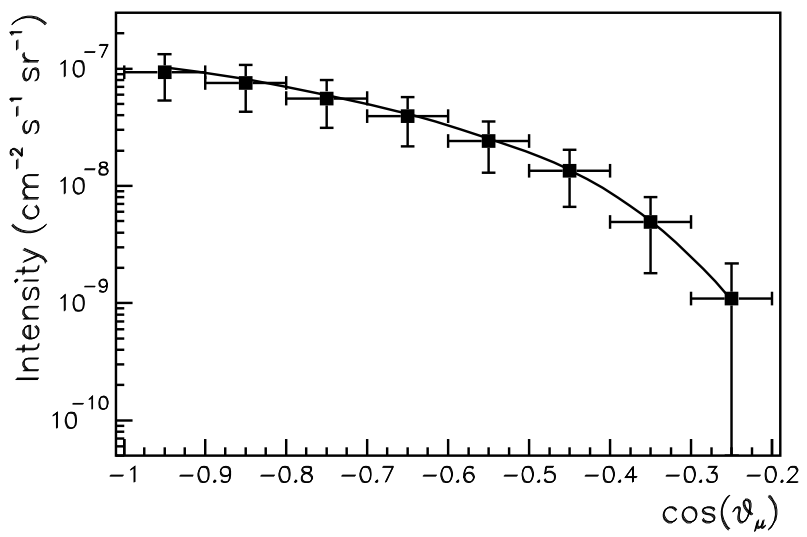

Fig. 16. Angular distribution of the atmospheric muon flux, $I\left(\vartheta_{\mu}\right)$, computed with Eq. 5, for the data acquired in the period 23-24 January 2007. The errors include statistical and systematic uncertainties added in quadrature. Data are compared with the simulated atmospheric muon flux (solid line).

Table 2

Systematic error: Contribution to the systematic error due to the uncertainty on each input parameter of the Monte Carlo simulation.

\begin{tabular}{lcc}
\hline \hline Input Parameter & Relative Uncertainty of the Parameter & $\Delta I / I$ \\
\hline$L_{a}$ & $\pm 10 \%$ & $+20 \%$ \\
$L_{b}$ & $\pm 10 \%$ & $-27 \%$ \\
PMT Quantum efficiency & $\pm 10 \%$ & $-19 \%$ \\
OM Angular Acceptance & $\pm 10^{\circ}$ & $+21 \%$ \\
\hline Total & & $-15 \%$ \\
\hline
\end{tabular}

The measured flux $I\left(\vartheta_{\mu}\right)$ was, then, transformed into muon vertical flux intensity $I\left(\vartheta_{Z}=0, h\right)$ using the formula:

$$
I\left(\vartheta_{Z}=0, h\right)=I\left(\vartheta_{Z}\right) \cdot \cos \left(\vartheta_{Z}\right) \cdot c_{c o r r},
$$

where the zenith angle is $\vartheta_{Z}=180^{\circ}-\vartheta_{\mu}$. The term $c_{\text {corr }}$ is a correction factor required for angles larger than $60^{\circ}$ as described in [39].

In Fig. 17 we show the Depth Intensity Relation for atmospheric muons. Results obtained by previous experiments are shown for comparison: MACRO [40] in standard rock, DUMAND [41], NESTOR [42], ANTARES [43,44]6] in

6 ANTARES has also recently presented a DIR curve based on a novel data analysis method [45], whose results are not quoted in Fig. 17, 
sea water, BAIKAL [46] in lake water, AMANDA [47] in ice. Results are also compared with the prediction of Bugaev et al. [48]. The NEMO Phase-1 data are in agreement with previous measurements and with Bugaev's prediction in the whole range of investigated depths.

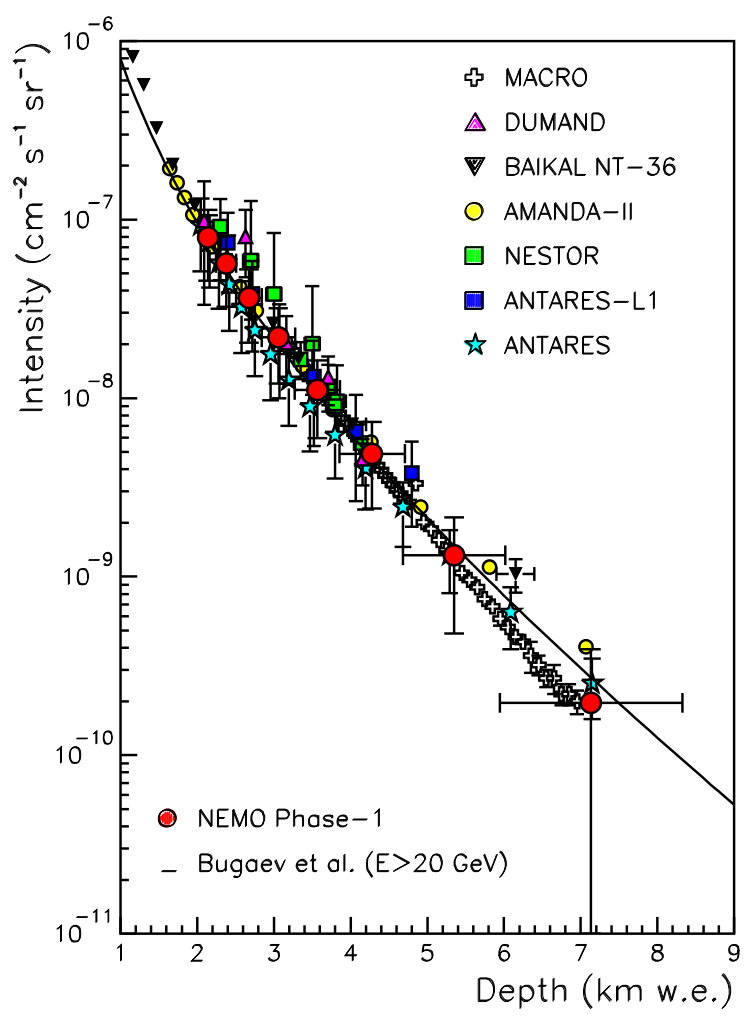

Fig. 17. Vertical muon intensity, $I\left(\vartheta_{Z}=0, h\right)$, versus depth measured using data acquired in the period 23-24 January 2007. For comparison, results from other experiments are quoted. The solid line is the prediction of Bugaev et al. [48].

\section{Conclusions}

The activities of the NEMO Collaboration have progressed with the achievement of major milestones: the realization and installation of the Phase- 1 apparatus.

With this apparatus it was possible to test in deep sea the main technological solutions developed by the collaboration for the $\mathrm{km}^{3}$ scale underwater neutrino telescope.

The angular distribution of atmospheric muons was measured and results were compared to Monte Carlo simulations. The vertical muon intensity was evalu- 
ated and compared with previous data and predictions, showing a good agreement.

\section{Acknowledgments}

We thank all INFN personnel that has contributed to the development and carry out mechanics, electronics and computing of the NEMO Phase-1 experiment. We also thank the ANTARES Collaboration for providing the detector simulation and track reconstruction codes, extensively used in this work. Eventually we thank our anonymous referee for useful comments and suggestions.

\section{References}

[1] F. Aharonian, Science 315-5808 (2007) 70.

[2] J. Albert et al., Astropart. Phys. 23 (2005) 493.

[3] F. Aharonian, et al., Nature 432 (2000) 75.

[4] V.A. Acciari et al., Ap. J. 684 (2008) L73.

[5] J.G. Learned, K. Mannheim, Ann. Rev. Nucl. Part. Sci. 50 (2000) 679.

[6] R. Wischnewski, for the Baikal Coll., Nucl. Instr. Meth. A 602 (2009) 14.

[7] R. Abbasi et al., Phys. Rev. D79 (2009) 062001.

[8] R. Abbasi et al., Ap. J. L. 701 (2009) 47.

[9] J.A. Aguilar et al., Astropart. Phys. 26 (2006) 314; Nucl. Instr. Meth. A 555 (2005) 132; Nucl. Instr. and Meth. A 570 (2007) 107; M. Ageron et al., Nucl. Instr. and Meth. A 578 (2007) 498; Nucl. Instr. and Meth. A 581 (2007) 695.

[10] P.A. Rapidis, Nucl. Instr. Meth. A 602 (2009) 54.

[11] NEMO web site: http://nemoweb.lns.infn.it.

[12] KM3NeT web site: http://www.km3net.org.

[13] G. Riccobene et al., Astropart. Phys. 27 (2007) 1.

[14] E. Migneco et al., Nucl. Instr. Meth. A 588 (2008) 111.

[15] ELETTRA Tlc web site: http://www.elettratlc.it/.

[16] E. Migneco et al., Nucl. Instr. Meth. A 567 (2006) 444.

[17] A. Aiello et at., NIM A (2009), accepted, DOI:10.1016/j.nima.2009.12.040. 
[18] C.A. Nicolau, for the NEMO Coll., Nucl. Instr. Meth. A 567 (2006) 552.

[19] G. Bunkheila, for the NEMO Coll., Nucl. Instr. Meth. A 567 (2006) 559.

[20] F. Ameli et al., IEEE Trans. Nucl. Sci. 55 (2008) 233.

[21] M. Ruppi, for the NEMO Coll., Nucl. Instr. Meth. A 567 (2006) 566.

[22] A. Rovelli, for the NEMO Coll., Nucl. Instr. Meth., A 567 (2006) 569.

[23] I. Amore, for the NEMO Coll., Nucl. Instr. Meth. A 602 (2009) 68.

[24] I.G. Priede et al., Deep Sea Research I 53 (2006) 1272.

[25] S. Escoffier, for the ANTARES Coll., Proc. of the $30^{\text {th }}$ ICRC, Merida, Mexico, July 3-11, 2007 arXiv:0710.0527 [astro-ph].

[26] Vallage B., for the ANTARES Coll., Nucl. Phys. B (Proc. Suppl.) 151 (2006) 407.

[27] F. Simeone, for the NEMO Coll., Nucl. Instr. Meth. A 588 (2008) 119.

[28] S. Galatà, 2008, Analisi dati e ricostruzione di tracce di muoni atmosferici con il rivelatore NEMO FASE-1, laurea dissertation, University of Catania, Catania.

[29] A. Heijboer, 2004, Track reconstruction and point source searches with Antares, PhD dissertation, Universiteit van Amsterdam, Amsterdam, The Netherlands (http://antares.in2p3.fr/).

[30] S. Aiello et al., Astropart. Phys. 28 (2007) 1.

[31] C. Distefano, for the NEMO Coll., Nucl. Phys. B (Proc. Suppl.) 190 (2009) 109.

[32] G. Carminati et al., Computer Physics Communications 179 (2008) 915; G. Carminati et al., Proc. of the 31st ICRC, Łódź, Poland, July 7-15, 2009 arXiv:0907.5563 [astro-ph.IM].

[33] Y. Becherini et al., Astropart. Phys. 25 (2006) 1.

[34] Y. Becherini, for the ANTARES Coll., Nucl. Instr. Meth. A 567 (2006) 477.

[35] G. Zech, Comparing Statistical Date to Monte Carlo Simulation: Parameter Fitting and Unfolding, DESY-95-113, June 1995.

[36] E. Andres et al., Astropart. Phys. 13 (2000) 1.

[37] G. D'Agostini, Nucl. Instr. Meth. A 362 (1995) 487; see also http://hepunx.rl.ac.uk/ adye/software/unfold/RooUnfold.html.

[38] P. Amram et al., Nucl. Instr. and Meth. A 484 (2002) 369.

[39] P. Lipari, Astropart. Phys. 1 (1993) 195.

[40] M. Ambrosio et al., Phys. Rev. D 52 (1995) 3793.

[41] J. Babson et al., Phys. Rev. D 42 (1990) 3613. 
[42] E. G. Anassontzis et al., in Proc. of the 23rd ICRC, Calgary, Canada, August, 1993; G. Aggouras et al., Astropart. Phys. 23 (2005) 377.

[43] M. Ageron et al., Astropart. Phys. 31 (2009) 277.

[44] M. Bazzotti, for the ANTARES Coll, Proc. of the 31 ${ }^{\text {st }}$ ICRC, Łódź, Poland, July $7-15,2009$.

[45] J. Aguilar et al., Astropart. Phys., (2009), accepted, DOI: 10.1016/j.astropartphys.2009.12.002, arXiv:0910.4843 [astro-ph.HE].

[46] I.A. Belolaptikov et al., Astropart. Phys. 7 (1997) 263.

[47] P. Desiati, for the AMANDA Coll. and K. Bland, in Proc. of the 28th ICRC, Tsukuba, Japan, July 31- August 7, 2003.

[48] E. Bugaev et al., Phys. Rev. D58 (1998) 05401. 